\title{
The Nested States Model: An Empirical Framework for Integrating Brain and Mind
}

\author{
George H. Denfield ${ }^{1,2,3}$ and Evan J. Kyzar ${ }^{1,2,3}$ \\ ${ }^{1}$ Department of Psychiatry, Columbia University, New York, NY, USA \\ ${ }^{2}$ Research Foundation for Mental Hygiene, Menands, NY, USA \\ ${ }^{3}$ New York State Psychiatric Institute, New York, NY, USA
}

\begin{abstract}
Philosophy of mind has made substantial progress on biologically-rooted approaches to understanding the mind and subjectivity through the enactivist perspective, but research on subjectivity within neuroscience has not kept apace. Indeed, we possess no principled means of relating experiential phenomena to neurophysiological processes. Here, we present the Nested States Model as a framework to guide empirical investigation into the relationship between subjectivity and neurobiology. Building on recent work in phenomenology and philosophy of mind, we develop an account of experiential states as layered, or nested. We argue that this nested structure is also apparent in brain activity. The recognition of this structural homology - that both experiential and brain states can be characterized as systems of nested states - brings our views of subjective mental states into broad alignment with our understanding of general principles and properties of brain activity. This alignment enables a more systematic approach to formulating specific hypotheses and predictions about how the two domains relate to one another.
\end{abstract}

\section{Introduction}

The cognitivist and connectionist paradigms that dominated cognitive science throughout the latter half of the 20th century defined much of the scope of contemporary neuroscientific investigation, with a consequent lack of emphasis on the direct investigation of subjectivity (Thompson, 2007; Gallagher, 2010, 2017; Taschereau-Dumouchel et al., 2022; Kyzar and Denfield, 2023). This lack of emphasis is all the more surprising given that this problem has significant practical import. Subjective experience is what we aim to temporarily prevent during anesthesia, what we need to identify after brain injury, and is what features predominantly in psychopathology.

In recent decades, a new and more firmly biologically-rooted theoretical perspective, known as the enactive approach, has arisen within philosophy of mind. Enactivism emphasizes the continuity between mind and life and returns the investigation of subjective experience to a place of central importance, drawing on the field of phenomenology to do so (Varela, Thompson and Rosch, 1991; Thompson, 2007). Enactivist accounts of mind highlight the reciprocal interaction between body and environment and the role of the organism as a self-organizing, autopoietic system engaged in participatory sense-making with the environment (De Jaegher and Di Paolo, 2007; Thompson, 2007; Di Paolo, 2018). This approach has been enormously influential across a 
number of domains including intersubjectivity (Di Paolo and De Jaegher, 2012), affectivity (Colombetti, 2017), and psychopathology (Fuchs, 2018).

Despite these successes and its natural links to biology, this promising theoretical perspective has not yet translated into an on-going, empirically-productive dialogue with contemporary neuroscience. This dearth of persistent cross-talk is not for lack of early efforts (Varela, 1996; Varela et al., 2001). Roadblocks remain in the quest for an interdisciplinary understanding of the mind, including the residual influence of cognitivism in neuroscience and the increasingly siloed nature of subfields within science and philosophy more generally. Additionally, the scope of enactivism as a full-fledged scientific paradigm is still under debate (Gallagher, 2017; Meyer and Brancazio, 2022). As Meyer and Brancazio (2022) note, "enactivism does not have a comprehensive story for how the various capacities of the mind can be explained, and how these can be examined empirically [...] nor do we see the kind of scientific 'divide and conquer' schemes for operationalisation taken up in cognitivist cognitive science."

Here, our overarching goal is to develop an operationalized scheme for the empirical examination of subjective experience in relation to neurophysiological processes. As enactivism takes a holistic view of the relationship between the entire organism and its environment, we are alert to avoid the mereological fallacy highlighted by Fuchs (2018) in which brain activity is mistakenly seen as sufficient to account for the mind. Nevertheless, the nervous system still plays a necessary and vital role, and delineating the full extent of this role is critical in understanding the link between biology and subjectivity. Indeed, the enactivist view that mind is a result of more than brain activity alone highlights all the more starkly the question of what exactly is the relationship between subjective experience and neurophysiological processes. Here, we present an empirical framework to address this question.

We begin by summarizing work in the phenomenological and enactive literature in the preceding decades describing a layered, or nested, quality of subjective experience. While numerous authors have more or less directly engaged with this notion, we are not aware of a dedicated, in-depth exposition of this viewpoint. We, therefore, provide such an account, developing what we call the Nested States Model (NSM). We show that experience can be characterized as a system of nested states, and we show how this nested structure constrains how one mental state flows into another. We then expound on what is, in our estimation, the principal advantage of the NSM - its ability to generate empirical predictions which can be tested using the methods of modern neuroscience. Specifically, we show that activity in the nervous system can also be described as a system of nested states, and we explore how to build out the bridge between the phenomenological and neurophysiological domains created by this recognition of structural homology. Given that both domains are seen to be systems of nested states, our view of subjective mental states is brought into broad alignment with our understanding of brain activity, allowing us to formulate specific hypotheses and predictions about how the experiential and neurophysiological domains relate to one another. In this manner, the NSM provides a targeted 'scheme of operationalization' (Meyer and Brancazio, 2022) from a phenomenological and enactivist vantage point that offers a systematic way to investigate subjective experience from the lens of both neuroscience and philosophy of mind. 


\section{Section I: A phenomenological analysis of the nested structure of experience Emerging views on the layered quality of experience}

Recent work in the enactive and phenomenological literature has begun to converge on a view of mental states and the structure of subjective experience which recognizes the layered quality of experience. We will describe and develop this view of mental states, showing that while it has primarily been applied to the study of affective and emotional states, the approach can be broadened and applies to the structure of experiential states in general. Importantly, we aim to render this layered, or nested, quality in terms that are empirically tractable.

An exposition of any experiential framework from an enactivist perspective would be incomplete without acknowledging the role of the lived body. While much of our later discussion will focus on empirically testing the relationship between subjective states and brain dynamics, we do not mean to remove the brain from its proper place - situated within a body. Quite the contrary, embodiment provides the ultimate foundation for experiential states. This point has been discussed at length in the enactivist literature, and the notion that a disembodied "brain-ina-vat" could somehow replicate lived experience is perhaps best refuted by Thompson and Cosmelli (2011). They argue that body and brain are dynamically entangled to the extent that any "vat" would essentially become a surrogate body. This line of reasoning demonstrates the indispensable nature of the lived body when discussing brain activity, and Thompson and Cosmelli (2011) state that it is precisely this embodiment that provides the background for creature consciousness, or the feeling of what-it-is-like-to-be a phenomenally conscious organism. Particularly notable for our purposes, Thompson and Cosmelli claim that this creature consciousness is intimately tied to background states of consciousness, such as arousal and the sleep-wake cycle, stating "background states of consciousness, such as wakefulness and dreaming, are domain general, not modality specific. They characterize one's overall phenomenal perspective as a conscious subject" and constitute "global modulations of creature consciousness." Thus, Thompson and Cosmelli discuss the foundational role of the body in enabling subjective experience and allude to a layered structure of experience (through use of words like "background").

The view that experience has a layered structure is most clearly articulated in recent work by Ratcliffe (2008) and Colombetti (2017), though there are precursors to this idea in earlier phenomenological work. Ratcliffe argues that we catch glimpses of this notion in Heidegger's discussions of affect and mood, which form the basis for Ratcliffe's own work on existential feelings. Ratcliffe interprets Heidegger to claim that "affective states [...] amount to a background sense of being situated in a world" that "shapes all our experiences, thoughts and activities" (Ratcliffe, 2008, p42) and which "is changeable in structure" (ibid, p52). Here, we already touch upon the two key themes that we will develop in more detail in this section and which will constitute the core claims of the NSM. First, is the notion of a "background" aspect of experience which can change over time and which implies also the notion of an experiential foreground. Hence, we see an initial sketch of the layered quality of experiential states. Second, is the notion that the experiential background "shapes" other aspects of our experience in some way. 
This second concept is hinted at by Heidegger and further elaborated in Ratcliffe (2008). Heidegger characterizes moods as what "make it possible first of all to direct oneself towards something" (Heidegger, 1988). Ratcliffe makes this notion more concrete through an example of an instance of fear. He notes that "before one feels afraid, one already has a sense of belonging to the world, of being in a situation in which one is afraid," which is thanks to the mood one finds oneself to be in (Ratcliffe, 2008, p49). It is through this particular mood that "one is attuned to the world in such a way that experiences of object-directed fear are possible" (ibid, p49). The implication here, which forms the basis for the second key claim of the NSM, is that different background senses of being in a world - different Heideggerian moods in this case - constrain, shape, or make possible certain other aspects of our experiential states.

Ratcliffe delineates the phenomenological category of existential feelings as follows: "an existential feeling is a background sense of belonging to the world, which structures all experiences" (ibid, p77). He clarifies that the moods which Heidegger discusses are existential feelings, but more colloquial uses of the term 'mood' do not necessarily reference the "allencompassing existential orientations" he means this category to signify; he notes that the term 'affect' would also be appropriate for this category. Continuing on this theme, Ratcliffe states that "when one experiences an emotion, one already finds oneself in the world. And the way in which one finds oneself in the world disposes one to certain kinds of emotional experience" (ibid, p40). Thus, different existential feelings not only operate in the experiential background, they also dispose us to different emotional experiences, constraining more foreground aspects of our experiential states.

Colombetti (2017) describes this structure of affective states and its consequences most clearly when she states that "moods appear to facilitate some emotional episodes more than others" and affect "the kinds of emotional episodes [an organism] is likely to enact" (p77-78). She also touches on the notion of a layered quality of experience more explicitly when discussing Ratcliffe's existential feelings, noting that existential feelings are "deeper" than specific emotions, and she further suggests that "the sense-making activity of the organism, its primordial affectivity" is even deeper still (ibid, p81-82). Colombetti describes primordial affectivity as a "capacity [of a living system] to be sensitive to what matters to [it]" and as "deeper' than the emotions and moods [...] in the sense that it grounds them or makes them possible" (ibid, p2).

We want to point out here a difference between Colombetti's uses of the term "deep" and the perspective on depth that we will advocate through the NSM. Her use of the term "deep" specifically in reference to primordial affectivity is not the sense of depth or layering to experience that the NSM describes; however, her use of the term to refer to the relationship between existential feelings and emotions is the sense of depth the NSM intends. We agree that primordial affectivity is foundational for and enables one to have certain types of (affective) experience, but it is not, strictly speaking, a layer in our experiential state as such. Rather, it is a property of a living system, and as such, it does not change over time. We argue that such change over time is an essential feature of all layers of an experiential state in the NSM framework. 
On the topic of depth, both Colombetti and Ratcliffe use the terms foreground and background to differentiate aspects of subjective experience, and it is worth taking a moment to discuss these terms more explicitly. Colombetti (2011) uses these terms when discussing how bodily feelings relate to emotional experiences. She speaks of bodily feelings coming to the "foreground of awareness," noting that a foreground feeling "comes into relief, it makes itself apparent, it asserts its presence" (Colombetti, 2011). In contrast, a background bodily feeling "does not 'stand out' and is not apparent, but it is still nevertheless felt;" elsewhere she states that background feelings "are not attended" (Colombetti, 2011). From these definitions, it is unclear if Colombetti intends to fully equate notions of foreground versus background feelings with attended versus non-attended feelings. Ratcliffe, too, discusses the experiential foreground and background, and it is not always clear whether these terms should be taken as synonymous with attended (foreground) versus present-but-not-attended (background) feelings, or whether a more specific structural claim is intended.

Drawing from the totality of their work on the topic (especially Ratcliffe, 2008 and Colombetti, 2017) it seems clear that both authors view affective experience as being layered, and they argue this point convincingly. What remains unclear is if they mean to use the terms foreground and background to refer to these different layers or if they have a different sense in mind - one that distinguishes different degrees of attention or awareness. As we will see, the NSM provides a more explicit and concrete structural definition for the use of these terms, where foreground and background states explicitly reference different layers of our experiential states.

Thus, we have seen how recent work in the phenomenological and enactive literature has honed in on two key features of affective states and emotional experience. First, that they are layered; and second, that deeper, or more background, states constrain what types of more foreground emotional states are likely to occur. The core of our phenomenological claim is that we agree, and we think that these ideas apply even more broadly, characterizing not only affective states but the structure and dynamics of experiential states in general. On this basis, we turn now to a discussion of the NSM framework, with the purpose of elaborating and clarifying this layered view of experience.

\section{Experience as a system of nested states}

From a phenomenological standpoint, the NSM makes two core claims:

Claim 1: The basic structure of our experience is a system of nested states.

Claim 2: This nested structure constrains, and therefore guides, how one mental state flows into another, thereby governing how our experience changes over time.

These claims can be illustrated by examining a hypothetical moment of lived experience. In this example, at moment $x$, John is walking outside on the sidewalk when he stubs his toe, feeling a sudden, strong pain. Imagine also that earlier that day, John and his girlfriend of several years had a big fight and are in the midst of a potential break-up - a situation which has been consuming much of John's attention, up to and including the moment when he stubs his toe. In 
examining John's mental state at this moment in time, though he may be most acutely aware of the pain in his toe at moment $x$, there is, thankfully, more to his experiential state than just pain.

Indeed, his pain occurs in the context of, or more specifically, is nested in, a background of emotions - sadness, anxiety and perhaps some fear (or, alternatively, happiness or relief if he is not particularly pleased to be in this relationship at this point in his life). All of these features are part of his current experiential state, but the important aspect to note is that these are not all simply co-occurring - there is a particular structure to these features.

This structure is what we refer to as nested. By nested we mean that some features of the mental state, labeled as the more "foreground" features, occur in the context of certain other features, labeled as more "background" features, which in turn occur in the context of yet other, even more "background" features, and so on. To make this description more concrete, in our example, the feeling of pain would be said to exist in the foreground relative to his emotional state of sadness and anxiety, which are more-background features, all of which occur in the yet-more-background context of a particular state of arousal, that of wakefulness (as opposed to sleep, for instance).

We can visualize this structure schematically in Figure 1. Here, we get a sense of the relationships between the various features or components of John's mental state and their positioning in the foreground to background continuum. It may be helpful to imagine the various levels of foreground to background features of a mental state as sections of a collapsible telescope, with the narrowest piece corresponding to the most foreground features of a mental state - thoughts, images, sensations - and the widest piece to the most background features, our state of arousal (pertaining to the sleep-wake cycle). The question of how many layers comprise

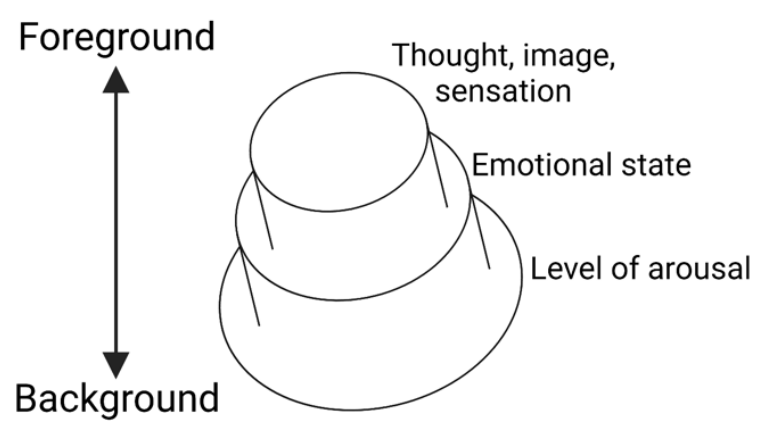

Figure 1. Example nested state configuration depicting aspects of John's experiential state at moment $x$. Each layer, or section of the collapsible telescope (see text), represents a different component feature of a mental state. our subjective experience will be discussed further below, and, we argue that in light of the framework here proposed, this is ultimately an empirical question. Our working example articulates only three layers for ease of conceptual illustration, but we argue below that there are more.

We can consider further variations on this example to help clarify this sense of the nested structure of experiential states. Imagine John and his girlfriend have not spoken in a few days, but he has heard from a friend that she is quite angry towards him. John wants to get a coffee from his favorite café, but this also happens to be his girlfriend's favorite café too. On the way there, John feels increasingly anxious and nervous about a potential encounter between the two of them. The thought occurs to him, "I wonder if she'll be there," then, "I hope she's not there." As he gets closer, he can't help but focus on their possible encounter and imagine various ways it may play out.

Focusing on the moment when John has the thought "I wonder if she'll be there," we would say that this thought is in the foreground, nested in a more background context of anxiety and 
nervousness, which itself still occurs in the context of wakefulness. We could imagine, though, that instead of actually being on the way to the café, John is simply dreaming about this scenario. In this case, the foreground features (the thoughts) and intermediate-background features (the emotions) of his mental state would be the same, whereas they would be occurring in a deeper background context of a level of arousal associated with sleep, likely REM sleep.

As is also apparent in the example above, our subjective experience is inherently temporal; hence, the metaphor of a stream of consciousness. The way in which subjective experience changes over time relates to Claim 2 of the NSM. To better understand Claim 2, we emphasize two key points. First, John's thoughts (foreground features of his mental state) change in the context of an emotional state (intermediate-background features) which does not change appreciably over this momentary time span. More accurately, the emotional state changes at a slower timescale than do his thoughts. Additionally, his state of arousal of wakefulness (a deeper background state) changes at an even slower timescale than his emotional state. Thus, we notice that different layers of features of the nested state change over different timescales. Second, in the context of his anxious mood, he felt that he could not help but focus on certain more-foreground thoughts. That is, the more-background features of a mental state exert a powerful influence on more-foreground features.

Thus, our notion of nesting entails more than just a relation between layers, it entails constraint. A foreground feature does not only occur in the context of a set of more background features, but those background features constrain which foreground features are likely to occur (as noted for emotion and mood by Ratcliffe, 2008 and Colombetti, 2017). This is the second key claim of the NSM. The degree to which, and manner in which, more-foreground features can change is constrained by which background features are present at a given point in time. That is, the foreground states that can be or are likely to be expressed at any given moment depend very strongly on the more immediate background states operating at that time, such that if background state A1 is active, then it is quite easy for foreground state A2 to occur but difficult for foreground state B2 to occur (which has a much higher probability of occurring if background state B1 is active). Figure 2 depicts this process of constraint across three nested layers, with hypothetical probabilities added to illustrate the point. 

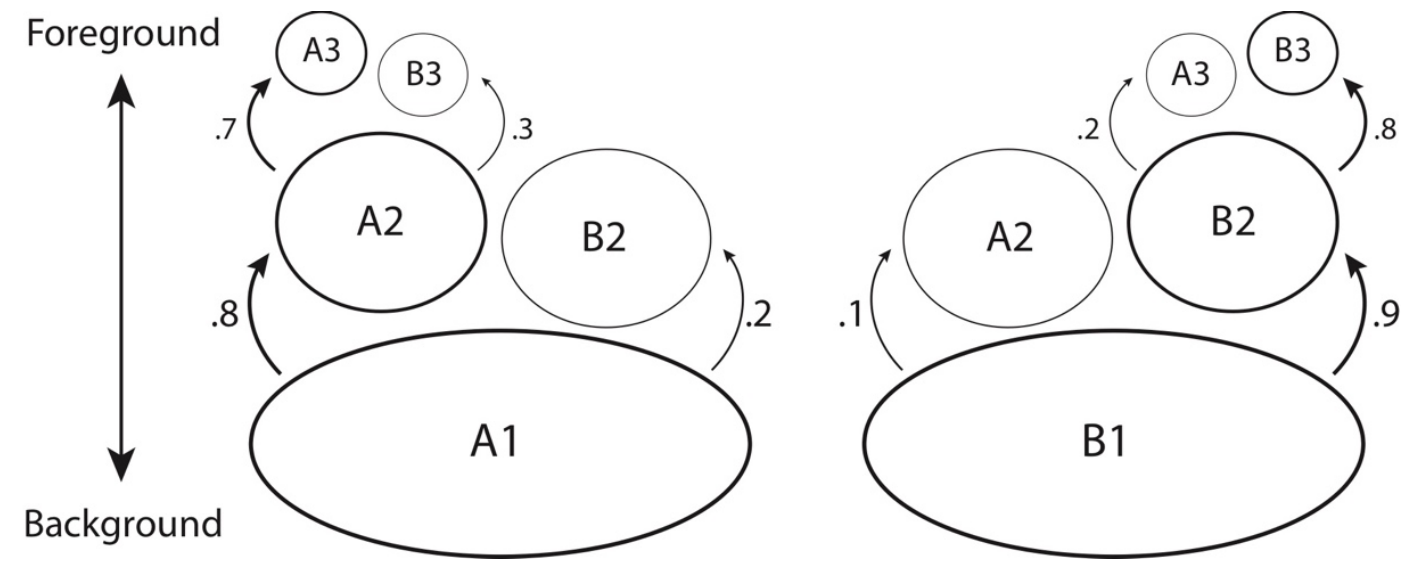

Figure 2. Background states constrain which more foreground states are likely to occur. On the left, we see that when background state A1 is active, the intermediate state A2 is much more likely to occur (with hypothetical probability 0.8 ) than is intermediate state B2 (occurring with probability 0.2). This process of constraint is recapitulated in the next layer, where foreground state A3 is much more likely to occur (with probability 0.7 ) than is foreground state B3, given that state A2 is active. On the right, background state B1 is active, making intermediate state B2 more likely to occur, which in turns makes foreground state B3 more likely than A3 to occur. Numbers in the figure depict hypothetical probabilities of a state occurring.

Given that he was anxious while on his way to the café, John was much more likely to have the specific, worried thoughts we saw in the above example. Nested in a context of anxiety, it was very unlikely John would have been fantasizing about meeting someone new at the café, for instance, rather than anticipating an unwanted encounter with his girlfriend. Alternatively, had John actually been unhappy in this relationship and relieved by the prospect of its ending, we would expect his thoughts would have been biased in a different direction, and perhaps, in that case, he would have been fantasizing about meeting someone new rather than focusing on an unwanted encounter. In this manner, background states constrain or sculpt the space of possibilities for which more foreground states may occur, shaping the riverbed for our stream of consciousness ${ }^{1}$.

Regardless of the specific thoughts John may have had on his way to the café, our example serves to illustrate the general point that background features constrain the foreground features of our mental states. This point can be made even more forcefully if we examine the most background features of our mental states. Take, for instance, the background state of arousal of slow wave sleep (contrasted with wakefulness). While this stage of sleep is critical for our mental functioning, it is generally not one in which we have any clear conscious experiences. That is, it is a background state which essentially precludes the occurrence of any sort of foreground features like thoughts, images, or feelings whatsoever.

To summarize thus far, we have developed the view of subjective experience as having a layered structure, elaborating this notion through the specific claims of the NSM and clarifying what is meant by an experiential foreground and background. Additionally, specifically through Claim 2, we see how the nested structure of a mental state guides how our subjective experience

\footnotetext{
${ }^{1}$ Ratcliffe (2008) also discusses existential feelings - background, world-disclosing orientations - as spaces of possibilities, akin to Husserl's notion of horizons.
} 
changes over time, partially accounting for the flow of the stream of consciousness. While we made use of a simple example to illustrate these claims - favoring conceptual clarity over taxonomic completeness - we end our treatment of the NSM from a phenomenological perspective by outlining an initial proposal for a taxonomy of nested states from an enactive standpoint.

\section{An initial hierarchical taxonomy of the NSM, and a note on causal directionality}

We feel that the principal benefit of the NSM is that it renders subjective phenomena into a structure which is empirically tractable. Using this heuristic organization, neuroscientific experiments can be designed to directly interrogate the various layers of experience, providing critical data which can then inform updated theorizing about the structure of mind and brain from both neuroscientific and phenomenological perspectives (aspiring to a process of reciprocal constraint a la Varela, 1996). With this sustained dialogue in place, the need for a hard-and-fast theory of the exact layers of experience is obviated, as we hope that repeated cycles of experimentation and the resultant updating of hypotheses will bring us closer to a unified understanding of the relationship between subjectivity and brain activity. We will explore the neuroscientific implications of the NSM in greater detail below. However, we first aim to sketch a proposal for an initial hierarchical taxonomy of the NSM.

We share the view put forward by Thompson and Cosmelli (2011), and others in the enactivist tradition, that the lived body is the vessel through which subjectivity flows - it provides the foundation for all other layers of the NSM. Indeed, we suggest that an enactivist hierarchy of nested states begins here, with the body as the deepest background state constraining all more foreground states. As discussed above, one feature of a state in the NSM framework is that it must be able to change over time, and more background states tend to change more slowly than more foreground states do. The body, barring the traumatic circumstances of sudden and severe injury, adheres to these requirements of a background state and provides the ultimate constraint on the more-foreground layers that can comprise our experiential state as a whole, in accordance with Claim 2 above. Nested within the background state of the lived body are several further layers, illustrated in Figure 3. Arousal states, or background states of consciousness comprising the sleep-wake cycle, appear next in our proposed hierarchy, followed by what we label motivational states. 
Motivational states include (at least) biological drives or needs and their occurrent feelings such as hunger, thirst, or lust, and possibly loneliness (in social species). There is a need for further phenomenological investigation of this aspect of our experiential states, and it is beyond the scope of the present essay to provide such treatment, but motivational states undoubtedly factor into our experiential states. ${ }^{2}$ They also act in accordance with Claim 2, influencing the types of moreforeground states which are likely to occur given a particular motivational state. For example, there is a colloquial American English term, being "hangry," which picks out a specific nested state configuration in which frustration and irritability are facilitated when in a state of hunger. We propose that motivational states are deeper in the hierarchy than existential feelings, as it is easier to imagine how states of hunger or thirst, for instance, may influence existential feelings than the reverse, but we emphasize this is ultimately an empirical question. To complete our proposal for an initial taxonomy, existential feelings are nested in the foreground of motivational states, followed next by emotional states, and in the foreground of all of this would be sensations, images, and thoughts. Again, our aim is for this taxonomy to serve as a starting point for an empirical approach to studying its relationship to brain dynamics - we expect that it will be clarified and revised further, through sustained dialogue between experimental and phenomenological

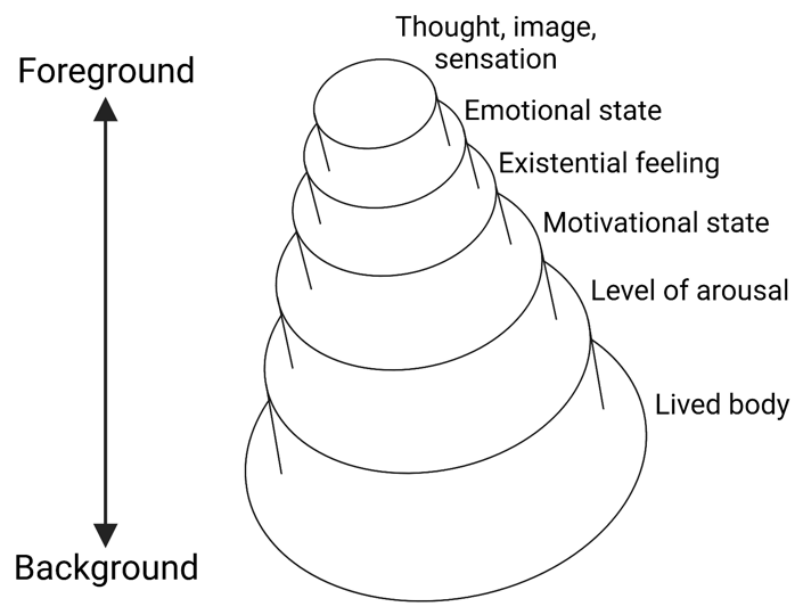

Figure 3. An initial hierarchical taxonomy depicting the various layers of an experiential state. investigation.

We now turn to the issue of causal directionality. In which direction do the levels of the NSM hierarchy exert influence? Our examples above principally describe a global-to-local, or background-to-foreground, causal directionality. Nonetheless, we can imagine that instead of walking along the sidewalk and stubbing his toe in the midst of an angst-ridden breakup, John was instead having a rather fine day prior to moment $x$. Then, immediately following his toe stub, the experience of pain, and of anxiety over any resulting injuries, leads to a more gradual change in his affect and existential feelings. In this manner, we see that the causal chain operates bidirectionally, in both a background-to-foreground and foreground-to-background fashion. Colombetti (2017) argues similarly when she notes that "the reiteration of certain emotion forms can carve a topology that leads to the relative stabilization of certain mood forms" over time (p78; see also Ratcliffe, 2008 and Fuchs, 2018 for similar arguments).

\footnotetext{
${ }^{2}$ We suspect that there are aspects of the notion of primordial affectivity that could be incorporated with several layers of our proposed hierarchy, including the body, motivational states, and existential feelings, particularly given Colombetti's references to Spinoza's conatus and Heidegger's moods.
} 
These bidirectional causal chains through layers of subjective experience are reminiscent of the ecological hierarchy identified by Fuchs (2018). He identifies levels of this hierarchy and their interaction with the environment; molecules interacting via metabolism, cells and tissues via homeostatic processes, and finally, the organism via perception and movement. Fuchs describes this hierarchical organization as a structuring influence, which constrains and integrates functions at other levels into emergent patterns. Plainly stated, both local-to-global and globalto-local influences constrain the possibility space in other parts of the hierarchy. This ecological model is extended to the subjective and, crucially, neuronal domains: "My thoughts-as agile as they may be-are never absolutely free. Rather they follow various paths which my experiences pre-draft as potential routes... the neuronal processes must also orient themselves along such pathways" (Fuchs, 2018, p234).

This line of reasoning raises the question, is the structure of subjective experience mirrored in the activity of the nervous system? We believe that it is. In the next section, we argue that activity in the nervous system shares a fundamental structural similarity with subjective experience; that it, too, can be described as a system of nested states. Indeed, we see this recognition of structural homology as the key insight of the NSM, leading to its most promising application - its potential to act as a bridging framework between phenomenological inquiry and neural data.

\section{Section II: The Nested States Model, from a Neurobiological Point of View Brain states exhibit a nested structure}

On a neurobiological level, we assert the same claims with regard to the dynamics of neuronal activity as were made for subjective experience.

Claim 1b: The basic structure of activity in the brain is a system of nested states.

Claim $2 b$ : This nested structure constrains how one brain state can flow into another.

There is a wide range of evidence from systems neuroscience that supports these claims that brain states share a homologous structure with experiential states. We will draw on this work to support the general validity of this perspective on brain activity, while highlighting clear initial points of overlap between recent studies on brain states and certain background states of the hierarchy we proposed in the previous section (Figure 3).

We can conceptualize a brain state in a similar manner as a mental state - as a snapshot of the activity occurring across the brain at a given moment in time. In neuroscience, such brain states are typically identified according to a set of measurable and quantifiable variables related to neuronal activity, such as the firing rate of each neuron being recorded, or the power in different frequency bands of the recorded local field potentials (LFPs) generated by the activity of groups of neurons. The state of the brain is then defined as the point in a multi-dimensional state space that these identified variables comprise (a mathematical form of the snapshot).

The study of brain states is an active field of research in systems neuroscience (for a review, see McGinley et al., 2015a). The earliest studies of brain states focused on characterizing the various states of arousal related to the sleep-wake cycle of mammals. Recall from the previous section that we identified arousal states, or states of consciousness comprising the sleep-wake cycle, as a deeper background layer of our experiential states (Figure 3). Analogously, we argue 
the neurophysiologically-defined sleep-wake states comprise deeper, background brain states in the hierarchy of nested brain states. These background sleep-wake states can be differentiated based on characteristic patterns of neuronal activity associated with each state, such as through measurements of the electric field potentials created by that collective activity (measured by scalp or brain-surface electrodes as an electroencephalogram [EEG] or by extracellular electrodes as LFPs). Indeed, these patterns are taken to define the various sleep-wake states, and three initial states were identified with these methods: the waking state, slow-wave sleep (SWS), and rapid eye movement (REM) sleep (Steriade et al., 1993; Destexhe et al., 1999; Steriade et al., 2001; Brown et al., 2012).

Gervasoni et al. (2004) distinguished these three sleep-wake states by constructing a 2dimensional state space with axes defined based on the LFP signals that were recorded from multiple forebrain areas in rats. The waking, SWS, and REM states occupied distinct regions in the state space constructed based on these LFP signals. Further, these states could all be reliably distinguished based on electrophysiological measures alone, without reference to behavioral variables such as movement. Importantly, these background brain states are mutually exclusive - only one state is active at a time.

Nested within these background brain states are specific, more-foreground brain states. McGinley et al. (2015a) discuss "sub-states" of the waking state, and the concept is similar to our notion of a foreground state. This recognition of a variety of sub-states of the waking state is a nascent area of research with no fully characterized taxonomy of layers or more-foreground states. One of the key applications of the NSM is that it provides the means to organize an approach within neuroscience (as well as within phenomenology, and between the two) to guide the study of such states and build out this taxonomy. As we discuss next, the initial research done in this direction provides further support for Claims $1 \mathrm{~b}$ and $2 \mathrm{~b}$.

Two such sub-states that have been relatively well-characterized in animal models are those of the active state, or active exploration (AE) state, and the state of quiet wakefulness (QW). From the standpoint of the NSM, these states constitute more-foreground brain states that occur nested in the context of the background waking state. Though they exhibit a variety of characteristic differences in brain activity patterns, these states can similarly be distinguished based on typical LFP patterns across the brain (McGinley et al., 2015). The AE and QW states can be said to exist on the same level of the nested hierarchy because only one or the other appears to exist at a given time. Further, the AE state is associated with specific behaviors such as whisking (the rhythmic, stereotyped movement of whiskers used to explore features of the environment) or locomotion, which do not occur in the QW state (McGinley et al., 2015). Given that whisking and walking would require different patterns of activity in motor cortical neurons (Ebbesen and Brecht, 2017), these behaviors can be said to index different yet-more-foreground brain states occurring within the AE state, which itself is nested in the waking state. Figure 4 provides a graphical depiction of this nested relationship.

We can see how such findings support Claim $2 \mathrm{~b}$ as well, which states that a given background state constrains which more-foreground states are likely to occur while that background state persists (see Figure 2). As a basic example, the AE state is only known to occur in the waking 
state, suggesting there is something about the SWS state that precludes the occurrence of the AE state (or at least makes it exceptionally unlikely to occur), while the waking state facilitates it. We can see a similar process of constraint in the next layer of this hierarchy when noting that whisking or locomotion do not occur in the QW state.

Recent work in zebrafish also describes two moreforeground brain states nested within the waking state. Marques et al. (2020) label these states as the exploitation and exploration states. Behaviorally, the exploitation state in zebrafish is one in which longrange movements associated with exploring an environment are suppressed, but specialized, shortrange movements related to hunting for prey are facilitated; while the converse is true for the exploration state (Marques et al., 2020). It is an intriguing and open empirical question whether the exploration state described in zebrafish could be said to be similar to the AE state we encountered above in mice, and, as we argue, this is exactly the type of question that the NSM both helps to highlight as meaningful and offers an approach towards answering.

By imaging activity across the whole brain of zebrafish in these two states, Marques et al. (2020) identify two opposing brain networks that are respectively more active in the exploration or exploitation state, thereby demonstrating two opposing brain states nested within the waking state in zebrafish. These brain states facilitate specific more-foreground brain states, which have functional advantages depending on the organism's current environmental context. For instance, Marques et al. (2020) demonstrated that neuronal activity in the exploitation-state network correlated with activity in the cerebellum, which mediates specific hunting-related movements, and was anticorrelated with other brain regions involved in facilitating exploratory movements and behaviors.

Marques et al. (2020) also raise the question of how hunger and satiety might interact with exploitation and exploration brain states and, specifically, how it might influence transitions between the two states. This question is well-suited to the NSM framework, as it is asking how a more-background state, in this case a motivational state related to hunger, constrains which more-foreground state is likely to occur or be active at a given moment. That is, one might predict that a low satiety background state would make the more-intermediate exploitation state more likely to occur, given that this is the state supporting hunting behaviors.

While Marques et al. (2020)'s data do not adequately address this question, a separate study by Allen et al. (2019) does demonstrate the profound role that deeper background motivational states have on constraining brain activity and which more-foreground states are likely to occur or persist. This study recorded the activity of over 20,000 neurons across 34 brain regions in the mouse while the mice performed a task for which they received a water reward. The authors compared both spontaneous and stimulus-related brain activity when mice were in a state of 
thirst or satiation. They found a widespread network of neurons whose activity corresponded to the motivational state of thirst or satiety, as well as significant changes in brain-wide activity patterns that depended on the motivational state of the organism (Allen et al., 2019). That is, they found that the way in which neurons across the brain responded to the presence of specific sensory stimuli was motivational-state-dependent, precisely in a manner that would be expected of a more-background-level brain state in the NSM framework.

To summarize the work reviewed thus far, we see that brain states exhibit a nested hierarchical structure, homologous to that of experiential states. Recent research lends support to the initial taxonomy proposed from a phenomenological perspective. Arousal, or sleep-wake, states feature as deeper background states in both brain state and experiential state hierarchies, and motivational states appear nested therein in both hierarchies as well. One question that arises at this point is how far does this correspondence go? We argue that it goes quite far, as we will discuss below, but the ultimate extent and degree of overlap between nested state hierarchies in both domains is an empirical question which the NSM both helps to frame and to address. Thus, in the remainder of this essay, we turn to a discussion of what this empirical approach looks like through the lens of the NSM.

\section{Section III: Bridging the Gap}

Let us, once again, frame the central problem on which the NSM helps us to get a meaningful hold. The problem is not that we lack methods to associate aspects of our subjective experience with certain neurophysiological processes. The methods of neurophenomenology provide such methods (Varela, 1996; Thompson et al., 2005; Thompson, 2007), and, indeed, they will factor prominently in the empirical approach we advocate. What we lack is a framework that brings our views of subjective mental states into broad alignment with our understanding of general principles and properties of brain activity, and with which we could begin to clearly hypothesize about and make specific predictions regarding how alterations in certain brain states might impact our subjective experience in a systematic way. The NSM provides this framework, which we view as its central contribution.

For instance, how do our motivational states interact with different existential feelings to bring about specific foreground aspects of our subjective experiences, such as particular thoughts? Absent the NSM, it is difficult to formulate this question so precisely. The NSM helps us to organize our thinking about this problem while simultaneously offering an empirical approach towards potential solutions. The questions of why we have the experiences we do at the particular moments we have them, and how brain activity might relate to and support these experiences, become questions of how nested state configurations relate to one another in each domain. With this broader goal in focus, we can see why the task of building out these hierarchies and examining their overlap is a central project within the context of the NSM.

In this section, we begin to sketch out what an empirical approach to bridging the subjective and neurophysiological domains looks like utilizing the NSM framework, with the recognition that this treatment is necessarily preliminary. We address three general sets of empirical questions, which we identify as domain-general, domain-specific, or cross-domain questions. Domain-general questions focus on properties of nested state hierarchies in general. For instance, how do we characterize such systems and their dynamics, or how do we determine at what level 
in the hierarchy a particular phenomenon should be placed? Domain-specific questions pertain to developing more complete state taxonomies, be they experiential or brain states. Lastly, crossdomain questions focus on clarifying the relation between experiential states and brain states, and here we anticipate that the methods of neurophenomenology would be especially useful.

From a domain-general standpoint, we have seen how both our experiential states and patterns of brain activity can be characterized as systems of nested states. In general terms, the NSM describes systems that exhibit a nested, hierarchically organized, context-dependent structure; hierarchical in that there are different levels of states of the system, ranging from background to foreground (Figures 3 and 5), and context-dependent in that each level of states acts as a context which constrains the way more-foreground levels of states can evolve (Figure 2). The methods of dynamical systems theory are well-suited to characterizing and analyzing such systems, particularly in regards to how they change over time (Freeman, 2000; Ižikevič, 2010; Colombetti, 2017; Favela, 2021). In the language of dynamical systems, background states partition the overall state space - the space of possible states the system could inhabit - into subspaces, restricting the range of foreground states a system may occupy. Thus, a background state can be understood as an attractor, which creates a tendency for the system to inhabit certain types of more-foreground states. Colombetti (2017) explicitly describes affective, or mood, states and their relation to emotional states in these terms. Many of the studies on brain states cited in the previous section make use of dynamical systems methods as well. Recall, Gervasoni et al. (2004) used state space analyses to distinguish various background states of wakefulness, and Allen et al. (2019) employed such methods to study motivational states.

Thus, we begin to see how to describe a system of nested states, but how do we determine the relative positioning of phenomena in a nested state hierarchy? We have already encountered two suggestions in our preceding discussion that help address this question, which we could label 1) an expressive exclusivity principle, and 2) a time-constant principle. Regarding expressive exclusivity, it appears that possibly-occurring states within a layer do not occur simultaneously with one another. For instance, we saw that either the AE or QW state is active at a given time point, and that they do not co-occur. If they were on different levels of the hierarchy, however, they could co-occur. Regarding the time-constant principle, we suggested that states in different layers of a nested state hierarchy change over different timescales - that more-background states tend to persist over longer timescales than do more-foreground states. ${ }^{3}$ Therefore, were we to note that a particular candidate-background state persisted (on average) for some longer time period relative to observed changes in other, candidate-foreground states, such a finding would suggest that we had correctly identified the state's relative hierarchical positioning as morebackground.

At the end of the last section, we asked how much further the correspondence between brain state and experiential state hierarchies goes. This question contains both domain-specific and cross-domain elements: 1) what layers follow motivational states in the brain state hierarchy, and 2) how do these layers correspond to those of the experiential state hierarchy in Figure 3? Drawing from our subjective state hierarchy, we might hypothesize that affective and emotional states are good candidates to follow motivational states on the neurophysiological side. However, the

\footnotetext{
${ }^{3}$ We emphasize that we are speaking of general tendency here, as there do seem to be instances in which states can transition quite quickly, as would be expected of dynamical systems.
} 
neuroscientific studies described above might suggest that the layer associated with AE/QW/exploitation states follows in the next-most-foreground. How does the NSM suggest we proceed to test these competing hypotheses?

An initial approach to answering this question would draw on the above proposed criteria for determining relative hierarchical positioning, which suggest that a comparison of the typical timescales, or time-constants, of affective and emotional states versus AE/QW/exploitation states might be helpful. Recent work on affective states in humans has begun to address this issue. Sani et al. (2018) recorded electrical activity from several brain areas associated with the limbic system. They found that specific patterns of brain activity, which were predictive of the subjects' reported moods (assessed by prompting the subjects' to report their mood states using a rating scale), varied on a timescale of 3.4 hours or slower. Kragel et al. (2016) studied the emergence of emotional episodes in human subjects, using models originally trained on whole-brain functional neuroimaging data to discriminate different emotional states that were evoked by film clips with music. They adapted these models to decode spontaneously occurring emotional states while subjects rested in an fMRI scanner and found that decoded emotional episodes varied over a timescale of minutes or less - shorter than the timescale of affective state variation from Sani et al. (2018). Kragel et al. also obtained subjective reports of mood states, using similar rating scales to Sani et al., and found that the frequency with which certain emotional states occurred was influenced by the subjects' reported mood states. For example, subjects who reported a more depressed mood exhibited a higher frequency of episodes of sadness, while subjects reporting a more anxious mood exhibited more episodes of fear, exactly as would be predicted through Claim 2 of the NSM. Thus, we find strong empirical support for the notion that emotional states are nested within the layer of affective, or mood, states. From the animal studies reviewed above, $\mathrm{AE} / \mathrm{QW} / \mathrm{exploitation}$ states tend to persist over a timescale of seconds to minutes, suggesting that they may constitute yet-more-foreground states (though we recognize that such comparisons should be done within species, and more work studying the correlates of AE/QW/exploitation states in humans is needed). Thus, affective and emotional states appear to follow motivational states in our initial brain state hierarchy. Indeed, we hypothesize that the layers identified in Figure 3, will have corresponding layers in the hierarchy of brain states (Figure 5). 


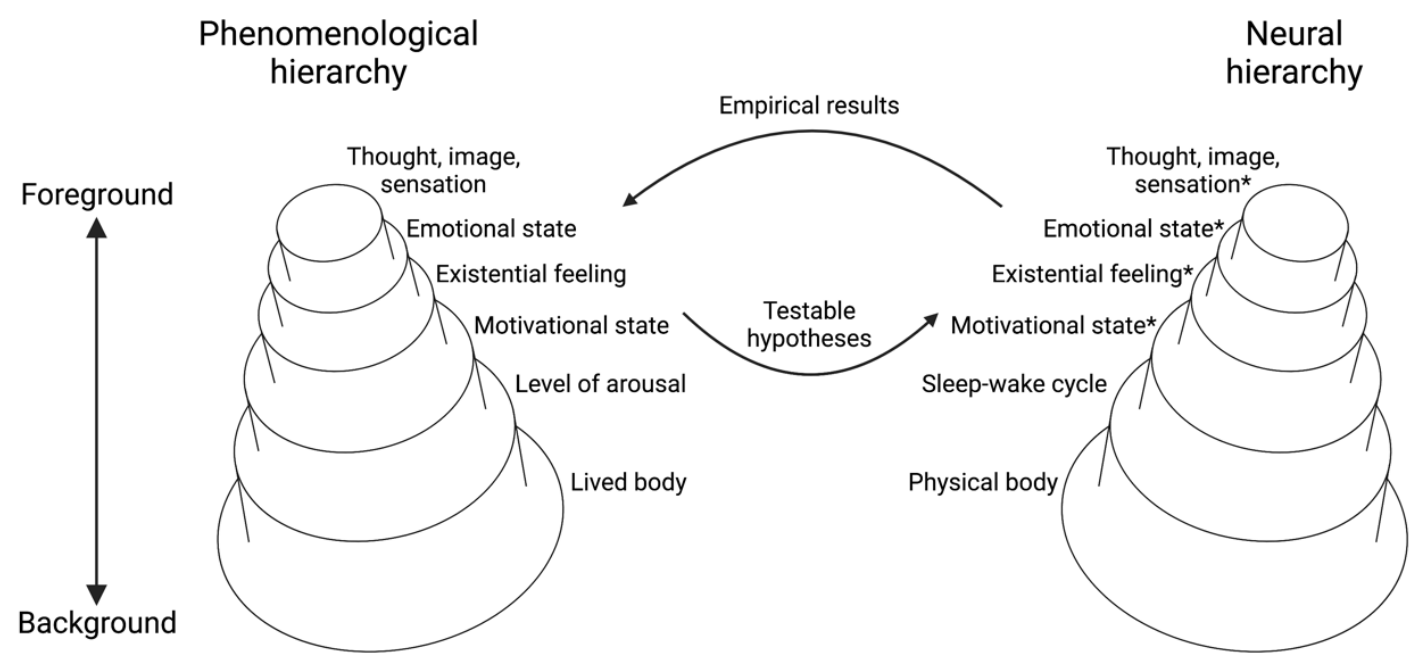

Figure 5. Proposed nested state hierarchies for experiential states (left) and brain states (right). We hypothesize that a layer in one hierarchy will correspond to a specific layer in the other. The specific levels shown here comprise an initial sketch - a hypothesis as to how these layers might be organized - which would be modified with ongoing phenomenological analyses and neural experimentation (as noted by the arrows in the center). While there is solid neurobiological evidence for neural states relating to the body and to the sleep-wake cycle, the exact correlates of the other four layers of the neural hierarchy are less clear (marked by an asterisk). Experiments of the variety proposed in this section would help to clarify further these hierarchies and their relation to one another.

Ultimately what we are most interested in is how nested state configurations in both domains relate to one another, which is the means by which we understand the relationship between subjective experience and brain states in the NSM. We've encountered several such cross-domain issues in the preceding sections, including calls for further phenomenological investigation of motivational states and the question of to which aspects of our experiential states the AE/QW/exploitation states might correspond. The studies of Sani et al. (2018) and Kragel et al. (2016) raise additional cross-domain questions and provide an opportunity to discuss how such investigation proceeds in the NSM framework. Though Sani et al. (2018) make frequent reference to "mood" states, they do not specifically define the term, and we are left with the question of to what aspect, or layer, of our experiential states their data corresponds. While their use of the term "mood" appears most consistent with what we defined above as affect or existential feelings, further phenomenological characterization of the probed affective states would have been helpful and should be incorporated in future studies. Similarly for Kragel et al. (2016). The methods of neurophenomenology are ideally suited for this pursuit, and indeed were developed for this very purpose (Varela, 1996; Thompson et al., 2005). Colombetti (2017) provides an excellent outline of how such methods could be incorporated into studies of affect, providing a general blueprint which could be readily expanded to explore all layers of the two proposed nested state hierarchies.

For instance, rather than using pre-specified rating scales, one could use phenomenologically richer methods, with more open-ended prompts, eliciting descriptions of subjects' mood and emotional states in their own words. Prompts could then be targeted more or less specifically to get at different aspects of emotional experience in the moment, possibly using short, semi- 
structured interviews to do so. The patient population from Sani et al. (2018) would have been ideally suited for such an approach, as they had intracranial electrodes in place for multiple days in a row, monitoring brain activity continuously, prior to surgery for epilepsy.

Ultimately, to more fully understand how nested state configurations in the subjective and neurophysiological domains relate to one another, such studies would need to be expanded to probe more than one layer of experience (and/or brain state) at a time. For example, future studies could incorporate manipulations of motivational states, while eliciting descriptions of the experience of those motivational states, of concurrent affective and emotional states, and of how they modify or change the experience of each other in combination. Such studies would provide rich insights into the relationships between our subjective experiences and the neurophysiological processes that support them. In the course of such studies we may discover that the positioning in the hierarchy of motivational states, say, relative to affective states needs to be adjusted. Such a hypothetical situation demonstrates the beauty of a useful empirical framework - it allows for and facilitates ongoing refinement based on observation without invalidating the framework itself. We envision that the NSM framework will support an iterative process of investigation, whereby phenomenological analyses inform and integrate with neuroscientific experimentation, with the ultimate goal of providing a 'scheme of operationalization' that will help forge a path to a unified understanding of mind and brain.

\section{Conclusion}

In our view, the primary contribution of the NSM framework is to make the study of how subjective states relate to neurophysiological processes empirically tractable through the recognition of a structural homology between the two domains. This recognition brings our views of subjective mental states into broad alignment with our understanding of general principles and properties of brain activity, allowing us to formulate specific hypotheses and predictions about how the two domains relate to one another, as we have just seen. Specifically, we have advanced the notion that both our experiential states and brain states can be described as systems of nested states. In this nested structure, more-background states facilitate the occurrence of certain more-foreground states, sculpting the riverbed for our stream of consciousness.

It is also worth remarking that though we have put forward an approach for beginning to unify the subjective and objective domains, this approach remains agnostic about the ultimate reducibility (or irreducibility) of subjective, mental phenomena. That we can develop a principled means of relating the two domains does not indicate how the reducibility question will be resolved one way or another, nor need this question be answered a priori for the approach here advocated to be feasible. Additionally, we view this framework as firmly rooted in the enactivist approach. Though this proposal focuses on brain activity, we do not mean to suggest that brain activity alone gives rise to mind and subjectivity. Ultimately, we hope that the NSM framework will provide a productive path forward through sustained, collaborative dialogue geared towards addressing the explanatory gap between biology and subjectivity. 
The Nested States Model

\section{References}

Allen, W.E. et al. (2019) 'Thirst regulates motivated behavior through modulation of brainwide neural population dynamics', Science, 364(6437), p. eaav3932.

Brown, R.E. et al. (2012) ‘Control of Sleep and Wakefulness', Physiological Reviews, 92(3), pp. 1087-1187.

Colombetti, G. (2011) 'Varieties of Pre-Reflective Self-Awareness: Foreground and Background Bodily Feelings in Emotion Experience', Inquiry, 54(3), pp. 293-313.

Colombetti, G. (2017) The feeling body: affective science meets the enactive mind. First MIT Press paperback edition. Cambridge, Massachusetts London, England: The MIT Press.

De Jaegher, H. and Di Paolo, E. (2007) 'Participatory sense-making: An enactive approach to social cognition', Phenomenology and the Cognitive Sciences, 6(4), pp. 485-507.

Destexhe, A., Contreras, D. and Steriade, M. (1999) 'Spatiotemporal analysis of local field potentials and unit discharges in cat cerebral cortex during natural wake and sleep states', The Journal of Neuroscience: The Official Journal of the Society for Neuroscience, 19(11), pp. 4595-4608.

Di Paolo, E. A. (2018) 'The enactive conception of life', in Newen, A. and Gallagher, S. (eds) The Oxford Handbook of Cognition: Embodied, Embedded, Enactive and Extended. Oxford University Press, pp. 71-94.

Di Paolo, E. and De Jaegher, H. (2012) 'The interactive brain hypothesis', Frontiers in Human Neuroscience, 6, p. 163.

Ebbesen, C.L. and Brecht, M. (2017) 'Motor cortex - to act or not to act?', Nature Reviews. Neuroscience, 18(11), pp. 694705.

Favela, L.H. (2021) ‘The dynamical renaissance in neuroscience’, Synthese, 199(1-2), pp. 2103-2127.

Freeman, W.J. (2000) 'Emotion Is Essential to All Intentional Behaviors', in M.D. Lewis and I. Granic (eds) Emotion, Development, and Self-Organization. 1st edn. Cambridge University Press, pp. 209-235.

Fuchs, T. (2018) Ecology of the brain: the phenomenology and biology of the embodied mind. First edition. Oxford, United Kingdom: Oxford University Press.

Gallagher, S. (2010) ‘Defining consciousness: The importance of non-reflective self-awareness', Pragmatics $\mathcal{E}$ Cognition, 18(3), pp. 561-569.

Gallagher, Shaun (2017) Enactivist Interventions: Rethinking the Mind. Oxford University Press.

Gervasoni, D. et al. (2004) ‘Global Forebrain Dynamics Predict Rat Behavioral States and Their Transitions', Journal of Neuroscience, 24(49), pp. 11137-11147.

Heidegger, M. (1988) Being and time. New York: Harper and Row.

Ižikevič, E.M. (2010) Dynamical systems in neuroscience: the geometry of excitability and bursting. First MIT Press paperback edition. Cambridge, Massachusetts London, England: The MIT Press (Computational neuroscience).

Kragel, P.A. et al. (2016) ‘Decoding Spontaneous Emotional States in the Human Brain', PLOS Biology. Edited by M. Rushworth, 14(9), p. e2000106.

Kyzar, E.J. and Denfield, G.H. (2023) ‘Taking subjectivity seriously: towards a unification of phenomenology, psychiatry, and neuroscience', Molecular Psychiatry, 28(1), pp. 10-16. 
Marques, J.C. et al. (2020) 'Internal state dynamics shape brainwide activity and foraging behaviour', Nature, 577(7789), pp. 239-243.

McGinley, M. et al. (2015) 'Waking State: Rapid Variations Modulate Neural and Behavioral Responses', Neuron, 87(6), pp. 1143-1161.

Meyer, R. and Brancazio, N. (2022) 'Putting down the revolt: Enactivism as a philosophy of nature', Frontiers in Psychology, 13, p. 948733.

Ratcliffe, M. (2008) Feelings of being: phenomenology, psychiatry and the sense of reality. Oxford ; New York: Oxford University Press (International perspectives in philosophy and psychiatry).

Sani, O.G. et al. (2018) 'Mood variations decoded from multi-site intracranial human brain activity', Nature Biotechnology, 36(10), pp. 954-961.

Steriade, M., Nuñez, A. and Amzica, F. (1993) 'A novel slow (<1 Hz) oscillation of neocortical neurons in vivo: depolarizing and hyperpolarizing components', The Journal of neuroscience: the official journal of the Society for Neuroscience, 13(8), pp. 3252-3265.

Steriade, M., Timofeev, I. and Grenier, F. (2001) 'Natural waking and sleep states: a view from inside neocortical neurons', Journal of Neurophysiology, 85(5), pp. 1969-1985.

Taschereau-Dumouchel, V. et al. (2022) 'Putting the "mental" back in "mental disorders": a perspective from research on fear and anxiety', Molecular Psychiatry, 27(3), pp. 1322-1330.

Thompson, E. and Cosmelli, D. (2011) 'Brain in a Vat or Body in a World? Brainbound versus Enactive Views of Experience':, Philosophical Topics, 39(1), pp. 163-180.

Thompson, E., Lutz, A. and Cosmelli, D. (2005) 'Neurophenomenology: An Introduction for Neurophilosophers', in A. Brook and K. Akins (eds) Cognition and the Brain. 1st edn. Cambridge University Press, pp. 40-97.

Thompson, Evan (2007) Mind in Life. Cambridge, Massachusetts: The Belknap Press of Harvard University Press.

Varela, F. et al. (2001) 'The brainweb: phase synchronization and large-scale integration', Nature Reviews. Neuroscience, 2(4), pp. 229-239.

Varela, F.J., Thompson, E. and Rosch, E. (1991) The embodied mind: cognitive science and human experience. 14. print. Cambridge, Mass.: MIT Press.

Varela, Francisco J (1996) 'Neurophenomenology: A methodological remedy for the hard problem', Journal of Consciousness Studies, 3(4), pp. 330-49. 